SPECIAL FEATURE

\title{
Child and adolescent injury as a result of falls from buildings and structures
}

\author{
J C Pressley, B Barlow
}

Injury Prevention 2005;11:267-273. doi: 10.1136/ip.2004.007724

Objective: To examine incidence, demographic risk factors, and patterns of injury resulting from falls from buildings and structures in areas with and without a legislation based prevention programme.

Design and setting: The Health Care Cost and Utilization Project (KID-HCUP) was used to produce national estimates of hospital admissions due to falls from buildings in the US. Areas of New York with and without window guard legislation were identified through the New York Statewide Planning and Research Cooperative System (SPARCS).

Subjects: Children and adolescents aged 0-18 years.

See end of article for

authors' affiliations

Interventions: Legislation based window fall prevention programme with enforcement.

Correspondence to: Dr J C Pressley, Columbia University, Mailman School of Public Health, 722 West 168th Street, Room 17-12, New York, NY 10032, USA; JP376@ columbia.edu

Main outcome measures: Hospitalization for injury as a result of falls from buildings and structures in areas with and without enforced mandatory window guard legislation.

Results: New York City has a higher proportion of the population residing in multifamily dwellings with 10 or more units compared with the nation $(53.8 \% v 12.6 \%, p<0.0001)$, but the incidence of injury resulting from falls from buildings is nearly half that observed in the US. For young children, warm weather risks begin earlier and extend later than previously reported. Incidence in very young minority children is nearly twice that of whites. Nearly $90 \%$ of children aged 0-4 years fall at home, but the proportion decreases linearly with age.

Conclusions: Window guards are associated with reduced injury resulting from falls from buildings and should be mandated in multifamily dwellings where small children reside. Prevention programmes for young children should be initiated in early spring and continued through fall.

A lthough injuries due to falls from buildings and other structures represent a small proportion of injuries to children and adolescents, many of these injuries are preventable through simple environmental modifications. ${ }^{1-4}$ Falls from height, originally documented as a problem in the northeastern US, particularly New York City, ${ }^{5-7}$ are now recognized as a cause of serious pediatric trauma on several continents. ${ }^{8-15}$ Falls from buildings, the majority of which are reported to involve a fall out of a window, may result in death or serious lifetime cognitive and physical disabilities. ${ }^{816-18}$ Traumatic brain injury, spinal cord injuries, major fractures, and psychological trauma are not uncommon. ${ }^{19} 20$ These injuries have been reported to cluster in resource limited urban neighborhoods where parental educational and income levels are frequently below the national average. ${ }^{15} 16$

Environmental interventions aimed at children were implemented in the cities of New York and Boston, resulting in a decrease in injuries due to this mechanism. ${ }^{1{ }^{34}}$ Injury reductions of up to $96 \%$ were reported after introduction of prevention strategies that included multifaceted interventional efforts with parental education, legislation requiring mandatory window guards in residences and common space of children aged 10 years and younger, and enforcement of that legislation. ${ }^{121}$ In this study, we examine demographic risk factors and characteristics of injuries as a result of falls from buildings and structures (which includes window falls) across the pediatric and adolescent age span, first in a national population hospitalized for this cause in the US, and then in New York City where legislated and enforced environmental modifications are in effect for children 10 years and younger.

\section{METHODS}

Data source(s)

The Kids Inpatient Database (KID-HCUP), provided the national sample of statewide acute care hospital discharges from 27 states for calendar year 2000. ${ }^{22}{ }^{23}$ KID-HCUP contains demographic, socioeconomic (median income for zip code of residence), diagnostic, treatment, and hospital outcome data on admissions. The cross sectional data set contains unweighted hospital discharge data on 2516833 pediatric admissions to community, non-rehabilitation hospitals (patients aged 19 years and less) and 7291032 weighted discharges. We excluded people aged 19 years of age because not all states reported on people older than 18 years. In addition, 61 routine, elective, or scheduled admissions for falls from buildings or structures were excluded as unlikely incident injury. For KID-HCUP, two states did not have data on month of discharge (Florida and Connecticut) and six states reported all races as other/unknown (Georgia, Kentucky, Maine, Oregon, Washington, and West Virginia). These states are excluded from incidence calculations by race and ethnicity, but appear in totals not stratified by race and ethnicity.

The New York Statewide Planning and Research Cooperative System (SPARCS), provided data at the zip code level for New York State residents hospitalized for the year 2001. Unlike KID-HCUP, SPARCS data, obtained from the New York State Department of Health, enabled us to examine the annual injury incidence specifically for New York City,

Abbreviations: CCS, Clinical Classifications Software; ICD-9-CM, International Classification of Diseases 9 clinical modification; KIDHCUP, Kids Inpatient Database-Health Care Cost and Utilization Project; SPARCS, New York Statewide Planning and Research Cooperative System. 


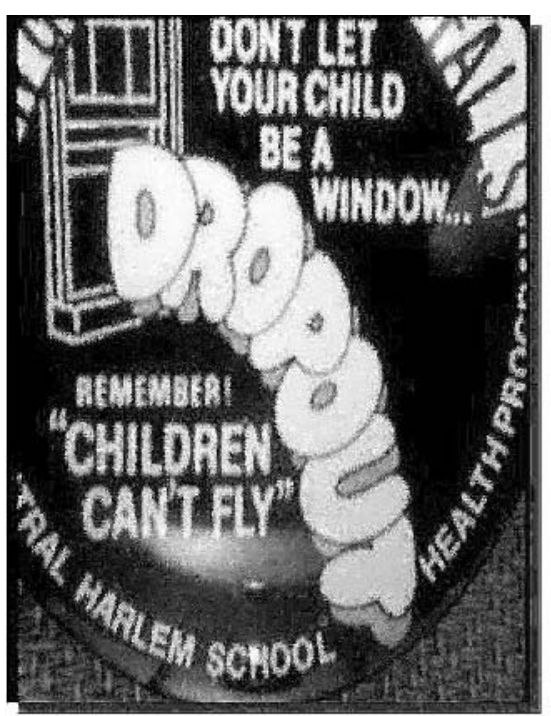

1972 "Children can't fly"

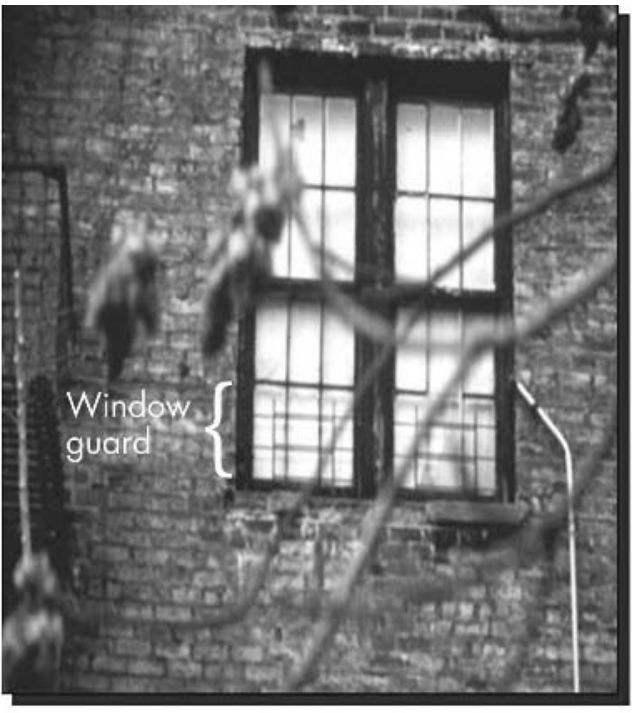

1979 Mandatory window guards
Figure 1 New York City Department of Health window fall prevention. which has a widely enforced window guard law. Approximately $40 \%$ of the children living in New York State reside in one of the five boroughs of New York City. The US census, conducted on the entire population decennially in the US, provided data on the population at risk by age, sex, and race/ethnicity and was used for the denominators in incidence calculations. The census collected number of units in housing structures using a categorical variable ranging from 1 to 50 or more. We used residence in a structure with 10 or more units as a proxy for exposure to multistorey dwellings. ${ }^{23}$

We defined acute injury to include urgent or emergent hospital admissions or admission through an emergency department resulting from falls from buildings or structures. We used external cause of injury (E-codes) from ICD-9-CM as well as classifications derived from the Clinical Classification Software (CCS) included as variables in the KID-HCUP data. ${ }^{24}$ ICD-9-CM code E882.0, used to identify falls from buildings or structures, excludes people who jump or fell from a building or structure to escape fire. In KIDHCUP, income is provided as a categorical variable at the neighborhood level using median income (in US dollars) for the zip code of patient residence. The definition of preadult disorders includes ICD-9-CM based codes for disturbances of emotions and conduct specific to or originating in childhood and adolescence. ${ }^{24}$

\section{Statistical analyses}

Cumulative incidence (per 100000 persons per year) was calculated for falls from buildings and structures for the US using weighted KID-HCUP (numerators), for New York City/ New York State using SPARCS data (numerators), and the US census 2000 (denominators). ${ }^{23}$ For analyses specific to New York City, we grouped the five boroughs of New York City (Bronx, Brooklyn, Kings, New York, and Richmond Counties), all of which are covered by window guard legislation. Multivariable logistic regression was used to identify independent factors and odds ratios for factors associated with falls from buildings. ${ }^{25}$ The "cases" for logistic regression were injuries resulting from falls from buildings/ structures and "non-cases" were children hospitalized for other reasons. The $\chi^{2}$ test was used in univariate analyses of categorical variables with statistical significance defined as less than or equal to 0.05 . Means are presented with standard deviations or with $95 \%$ confidence intervals. We used SAS 9.0 for processing the large databases. ${ }^{26}$

\section{RESULTS}

Of the 2163402 people age 18 years and younger who were discharged from a US hospital during the year 2000, 1161 discharges were classified as acute injury due to falls from buildings and structures. Of these, 70 were classified as intentional falls or jumps and 1091 discharges were categorized as unintentional incident injury due to falls from buildings or structures.

\section{Intentional self inflicted injury}

Of the 70 children and adolescents classified as having an intentional fall or jump, 27 (38.6\%) were whites, 10 (14.3\%) were blacks, 11 (15.7\%) were Hispanics, and 22 (31.4\%) were of other or unknown race or ethnicity. The age distribution of persons attempting suicide included $50(71.4 \%)$ people aged $15-18$ years, and $16(22.9 \%)$ aged $10-13$ years. The majority were in households earning incomes of $\$ 35,000$ or more $(55.2 \%)$. Comorbid mental health or alcohol or substance abuse diagnoses were reported for $57(81.4 \%)$ of those attempting suicide by this mechanism. Mental illness diagnoses included affective disorder in $16(22.9 \%)$, substance abuse in $12(17.1 \%)$, and other conditions in 44 $(62.8 \%)$. Of the 31 attempted suicides where place of injury was reported, nearly half $(45.2 \%)$ occurred at home and two thirds occurred during weekdays. Injuries categorized as attempted suicide are excluded from further analysis.

\section{Unintentional injury}

There were race/ethnic differences in the age distribution, income levels and place of injury for the 1091 admissions for unintentional falls from buildings (table 1). Among blacks and Hispanics, nearly half of hospitalizations occurred in the youngest age group, compared with one third in whites (table 1). Being male was associated with increased incidence of injury accounting for more than two thirds of all falls from buildings and structures. Falls among blacks clustered in communities with zip codes below the national median while most falls among whites were associated with incomes higher than the national median (table 1). Nearly $90 \%$ of very young children aged $0-4$ years for whom place of injury 


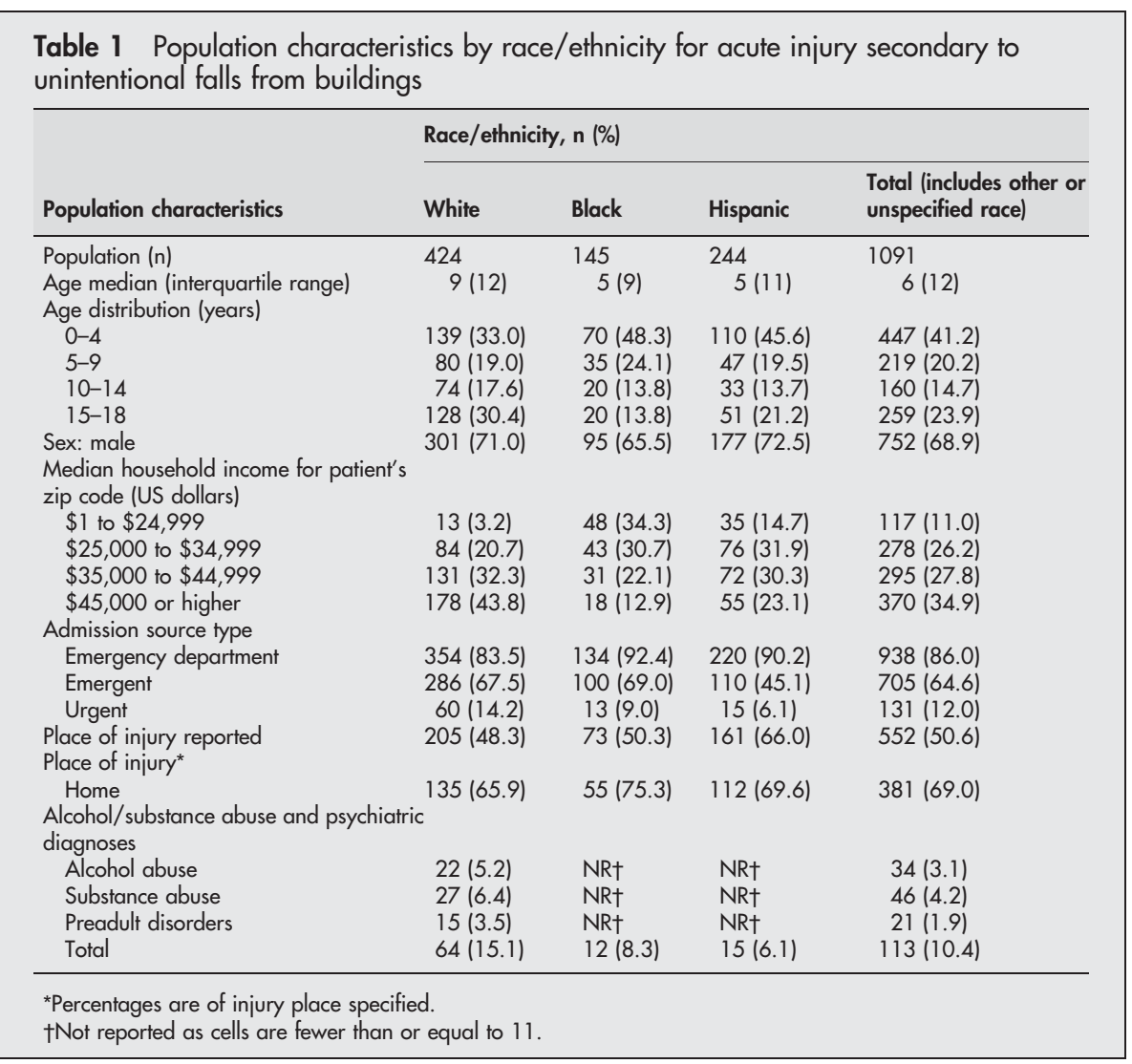

was reported fell at home (fig 2). The number of falls at home showed a linear decrease with age.

\section{Race and ethnic differences in acute injury incidence due to falls from buildings}

The cumulative incidence of injury due to falls from buildings is shown by age and race/ethnicity in table 2 . Among the total population, children aged $0-4$ years had a higher incidence than older children, although the pattern tended to be Ushaped across the age groups examined. Incidence among minority children aged $0-4$ years was approximately double that of similarly aged white children. Twelve patients (1.1\%) who lived to be admitted to the hospital died before discharge. Inhospital mortality did not differ by race/ ethnicity.

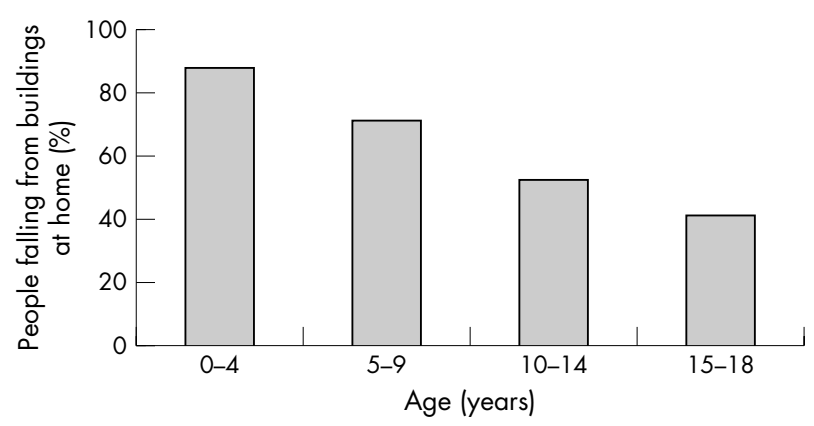

Figure 2 Unintentional falls from buildings and structures occurring at home by age. Percentages were calculated by obtaining the number of falls from buildings and structures with E-code 849.0 (injured at home) recorded in the hospital discharge data and dividing it by the number of people with any E-code recorded for place of injury.

\section{Incident injury in interventional $v$ non-interventional areas}

New York City had a higher proportion of the population residing in multifamily dwellings with 10 or more units compared to the nation $(53.8 \% \vee 12.6 \%, \mathrm{p}<0.0001)$, but the incidence of injury from falls from buildings was nearly half that observed in the US. Of the 30 falls involving children residing in New York City, approximately half occurred outside the age range covered by window guard legislation. Although incidence for the State of New York was lower than the national average $(2.47 \vee 2.81$ per 100000 people), this was due to lower incidence in New York City as incidence rates in areas of the state not covered by legislation were slightly higher than the national average (fig 3). Boston, Massachusetts and Chicago, Illinois implemented nonlegislative based intervention programmes. Although

Table 2 Estimated cumulative incidence of emergent and urgent hospital admissions for unintentional falls from buildings or structures by age and race/ethnicity*

\begin{tabular}{lllll}
\hline \multirow{5}{*}{ Age (years) } & \multicolumn{3}{l}{ Race/ethnicity } & \\
\cline { 2 - 5 } & White & Black & Hispanic & Totalt \\
\hline $0-4$ & 2.72 & 4.82 & 5.48 & 4.6 \\
$5-9$ & 1.23 & 2.1 & 2.4 & 1.98 \\
$10-14$ & 1.01 & 1.33 & 1.91 & 1.46 \\
$15-18$ & 2.74 & 1.52 & 3.38 & NA $\ddagger$ \\
Total & 1.87 & 2.42 & 3.37 & 2.81 \\
\hline
\end{tabular}

*Cumulative incidence (per 100000 people/year) for children aged 0-18 years hospitalized for falls from buildings or structures. Data source is KID-HCUP. Incidence is for acute hospitalized injury and does not include emergency department visits that did not require hospitalization or patients who died before being hospitalized. tIncludes other and unspecified races.

$\ddagger$ Not reported. Category has large percent unknown. 
KID-HCUP did not contain data for Illinois nor separate data for Boston, unintentional falls from buildings and structures for people aged $0-18$ years in the State of Massachusetts tended to be higher than for New York State (3.00 $v 2.47$ per $100000)$ and the US (3.00 $v 2.81$ per 100000$)$ (fig 3 ).

\section{Types of unintentional injury}

Types of injury sustained during falls from buildings were markedly different among the age groups (table 3 ). Multiple trauma was more common among teenagers aged 15-18 years than among young children and adolescents $(p<0.001)$. Very young children had high incidence of intracranial injuries and fractures of the skull and face, but had relatively infrequent spinal cord injury and fracture of the extremities in comparison with older children. Upper extremity fractures were less frequent in very young children but doubled or tripled in older children and adolescents. Lower extremity fractures were relatively uncommon in very young children, but increased with age, quadrupling in adolescents aged 15-18 years compared with children aged $0-4$ years. Fewer blacks had diagnoses of fracture than whites or Hispanics $(p=0.002)$. Mental health diagnoses were associated with higher incidence of spinal cord injury $(p=0.0001)$, and tended to be higher in those experiencing multiple trauma $(\mathrm{p}=0.057)$.
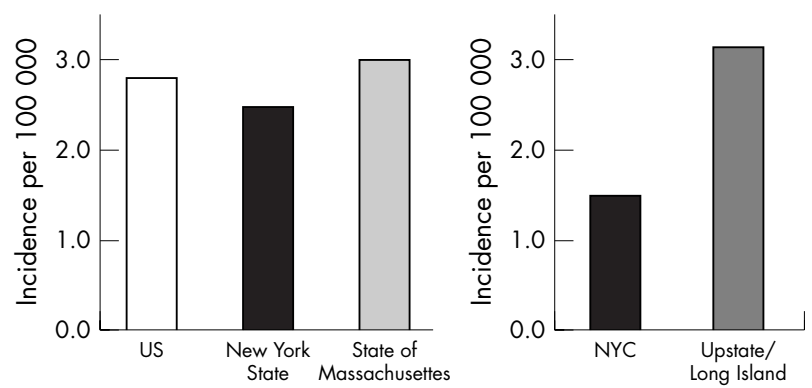

Figure 3 Cumulative incidence of emergent and urgent hospitalization admissions due to falls from buildings/structures in the US, New York State, Massachusetts, and regions of New York with and without legislation. Incidence is for people aged 0-18 years and does not include those treated and released in an emergency department, those not seeking treatment, or who died before admission to a hospital. US national incidence is estimated using weighted KID-HCUP data. The incidence for New York State, New York City, and Upstate/Long Island New York are from SPARCS.

\section{Seasonal unintentional injuries}

Injury due to falls from buildings showed seasonal gradients that varied across race/ethnic and age groups. In the total population, injuries began to increase slightly in early spring and began to decline in September (fig 4A). Injuries were lowest between the cold weather months of November and February (fig 4A). Seasonal patterns in very young children showed low frequency of injury in the winter (fig 4B). In contrast, older children and teenagers showed a more flattened seasonal distribution (fig 4C). There were notable race/ethnic differences, with Hispanics showing a tendency toward an extended "season of risk". In logistic models stratified by race/ethnicity for children aged 0-9 years, increased odds ratios were observed for an extended season of "warm weather" months that began in April and extended through September.

\section{Psychiatric, alcohol, and substance abuse diagnoses in those with unintentional injury}

Race/ethnic, socioeconomic, and age differences were noted for mental health, alcohol abuse, and substance abuse diagnoses. Mental health diagnoses were uncommon in very young age groups, but increased with age. Mental health diagnoses, including substance abuse, were highest in older adolescents aged $15-18$ years $(p<0.0001)$. Concomitant mental health or substance abuse diagnoses occurred twice as frequently in whites than in blacks or Hispanics $(p=0.015)$. Alcohol abuse diagnoses were an independent predictor of injury due to unintentional falls from buildings only in white adolescents (OR $=2.50,95 \%$ CI 1.60 to 3.90$)$.

\section{Race/ethnicity and socioeconomic status}

The distribution of socioeconomic status as measured using median household income for the patient's zip code of residence is shown in table 1 . In an attempt to disentangle socioeconomic status and race/ethnicity, separate models were developed for whites, blacks, and Hispanics. Among black children and adolescents, 35.5\% of whom lived in households earning $150 \%$ of the poverty level or less, a model of independent predictors of falls from buildings showed lower neighborhood income to be a significant risk factor only for the lowest income group in reference to the highest $(p=0.038)$. However, in separate models for whites and Hispanics, where only $3 \%$ and $10 \%$, respectively, lived at this income level, the neighborhood income results were mixed.

\begin{tabular}{|c|c|c|c|c|c|}
\hline & \multicolumn{5}{|l|}{ Age (years) } \\
\hline & $0-4$ & $5-9$ & $10-14$ & $15-18$ & All ages \\
\hline \multicolumn{5}{|l|}{ Types of injury, $\mathrm{n}(\%)$} & 1091 \\
\hline Multiple trauma & $27(6.0)$ & $\mathrm{NR}^{*}$ & $\mathrm{NR}^{*}$ & $32(12.4)$ & $78(7.1)$ \\
\hline Intracranial injury & $169(37.8)$ & 68 (31.1) & 30 (18.8) & $59(22.8)$ & $326(29.9)$ \\
\hline Spinal cord injury & $\mathrm{NR}^{*}$ & $\mathrm{NR}^{*}$ & $\mathrm{NR}^{*}$ & $20(7.7)$ & $26(2.4)$ \\
\hline Crushing/internal injury & $46(10.3)$ & 26 (11.9) & $14(8.8)$ & 35 (13.5) & $121(11.1)$ \\
\hline Fractures & $189(42.3)$ & $112(51.1)$ & $111(69.4)$ & $191(73.8)$ & $603(55.3)$ \\
\hline Skull and face & $104(23.3)$ & $28(12.8)$ & $14(8.8)$ & $20(7.7)$ & $166(15.2)$ \\
\hline Upper extremity & $50(11.2)$ & $61(27.9)$ & $58(36.3)$ & $65(25.1)$ & $234(21.4)$ \\
\hline Lower extremity & $32(7.2)$ & 25 (11.4) & $42(26.3)$ & $84(32.4)$ & $183(16.8)$ \\
\hline Neck of femur & $\mathrm{NR}^{*}$ & $\mathrm{NR}^{*}$ & $\mathrm{NR}^{*}$ & $\mathrm{NR}^{*}$ & $20(1.8)$ \\
\hline Other & $15(3.4)$ & $13(5.9)$ & $22(13.8)$ & $83(32.1)$ & $133(12.2)$ \\
\hline \multicolumn{6}{|l|}{ Open wounds } \\
\hline Head, neck, trunk & $58(13.0)$ & $24(11.0)$ & $12(7.5)$ & 29 (11.2) & $123(11.3)$ \\
\hline Extremities & $\mathrm{NR}^{*}$ & $\mathrm{NR}^{*}$ & $\mathrm{NR}^{*}$ & $16(6.2)$ & $36(3.3)$ \\
\hline
\end{tabular}



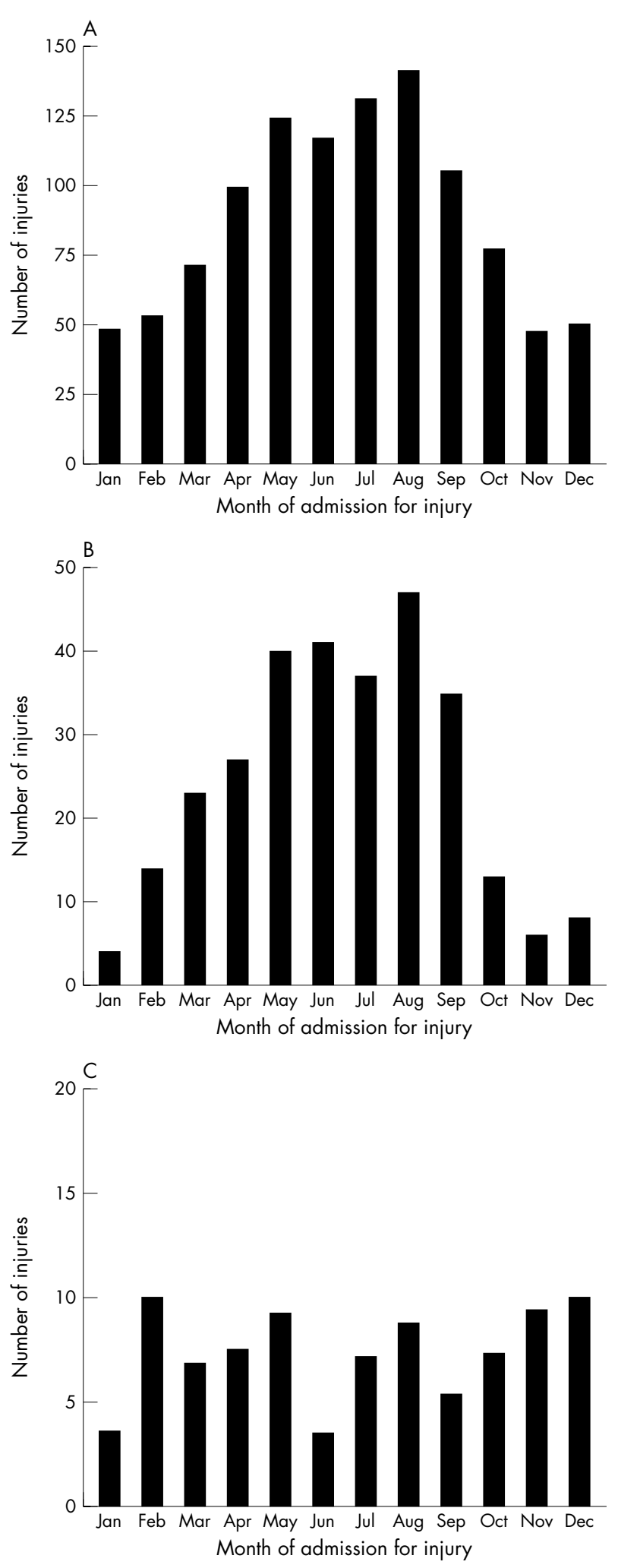

Figure 4 Seasonal distribution of injuries due to unintentional falls from buildings presented as the number of injuries by month of hospital admission for the total population (A), for children aged 0-4 years (B), and for adolescents aged $15-18$ years (C).

\section{Cost of hospital care associated with falls from buildings}

Median per person hospital charges for injuries from falls from buildings or structures were $\$ 8968$ (mean $\$ 17,020$ (SD
$\$ 28,576)$ ) (in year 2000 US dollars). Hospital charges were similar across age groups except in the oldest group where there was an increased tendency toward multiple trauma. Median charges for people with multiple trauma versus without were \$20,312 (mean \$40,388 (SD \$54,393)) and $\$ 8485$ (mean $\$ 15,253$ (SD $\$ 24,760)$ ), respectively. Length of hospital stay varied similarly, with hospital stay for those with versus without multiple trauma experiencing longer hospital stays (6.9 (SD 6.7) days v 3.3 (SD 5.6) days).

\section{DISCUSSION}

The risk of unintentional falls from buildings was bimodal, with the highest incidence in very young children aged 0-4 years and in teenagers. Among minority children, both Hispanics and blacks were at greater risk for injury from falls from buildings than white children. Risk factors varied significantly over the age span, with alcohol abuse/psychiatric diagnoses rising in importance in white adolescents and teenagers.

Consistently strong peaks in numbers of injuries have been reported to occur during warm weather months, generally reflecting increased falls from windows left open for home temperature regulation. ${ }^{12} 19$ Our findings mirror those of other studies when all ages are considered, however, significant differences emerge when age groups are examined separately. Our observation showed age related risks with higher warm weather risks for very young children and higher year round risk in adolescents. Seasonal differences were most pronounced in very young children, but flattened with increasing age, reflecting an increased tendency toward year round injury among 15-18 year olds. This pattern was also associated with a gradual shift in place of occurrence and risk factors for injury from falls from buildings. Changes in the age profile are probably multifactorial with older adolescent injury likely reflecting increased social influences, mental illness, and substance abuse. Although falls from buildings in younger versus older children likely represent a different sequelae of events requiring a different approach to effective prevention, the presence of window guards may deter both.

Our study found high psychiatric illness in intentional falls and a high proportion of substance or alcohol abuse in white teenagers experiencing unintentional falls. These findings are consistent with the findings of Gill, whose toxicological investigation of fatal falls from height in New York City documented similarly high incidence of use of psychiatric medication in suicide attempts and found that $36 \%$ of "accidental" fatal injuries due to falls from height were associated with use of alcohol or illicit substance. ${ }^{27}$ Although it appears likely that our unintentional falls may include some undiagnosed suicide attempts, unintentional injury is also a plausible explanation for falls secondary to impaired judgment associated with alcohol and drug abuse. Among suicide attempts by jumping from a building or other structure, nearly half occurred at home suggesting the possibility that window guards might also deter or divert home suicides through increased obstacles to use of this mechanism.

A window guard law, with annual enforcement, has been effective in New York City where exposure to high rises associated with multifamily dwellings is higher than the national average, but where incidence of injury due to falls from buildings is far lower. Despite declines in the early seventies, falls from height continued to be reported as a problem in New York in 1975 with one report noting as many as 159 falls and 19 deaths due to falls. ${ }^{28}{ }^{29}$ In 2001, 30 children were hospitalized as a result of falls from buildings and structures in New York City, but there were no reported window fall deaths and many falls were among older 


\section{Key points}

- Minority children are at a greater risk of injury from falls from buildings and structures than white, nonHispanic children.

- The season of risk for window falls extends longer than previously reported.

- The warm weather peak previously reported is observed in very young children in contrast to the more even year round risk observed in adolescents.

- Areas with versus without legislation have half the incidence of falls from buildings and structures despite having a more than fourfold higher proportion of multifamily housing.

- Diagnoses of mental illness and alcohol abuse were observed risk factors in white, but not minority adolescent and teenage falls from buildings and structures.

adolescents not covered by the law..$^{31}$ Although declines were noted following introduction of legislation, steeper declines were noted with enforcement of that legislation which included legal action with criminal charges against landlords failing to comply with the window guard law and with continued efforts to educate parents and consumers through a programme called "Children Can't Fly". ${ }^{36}$ Although prevention efforts have produced improvements in the absence of well designed legislation with concomitant enforcement, such areas report that nearly $40 \%$ of injuries resulting from falls from heights continue to be from window falls. In one state where educational campaigns were introduced in their largest city without mandatory legislation, we observed state level hospitalization incidence of falls from buildings and structures to be similar or higher than the national average although the city reports lower injuries from windows falls. ${ }^{4}$ We did not have information that allowed us to differentiate injury as a result of window falls from falls from buildings or to identify areas of this state reached by educational campaigns.

This study had limitations. Most importantly, the ICD-9CM code used to identify falls from buildings and structures includes non-window falls from buildings, such as falls from fire escapes. Although the data set was designed for such purposes, we made national estimates based on data from 27 states. Bias may have been introduced if the 27 states were not representative of the US population. For New York State, incidence produced using KID-HCUP and SPARCS were virtually identical. We were unable to include in our incidence calculations injuries in people dying before hospital admission, not seeking treatment, failing to receive the appropriate E-code, or those treated and released from an emergency department. Furthermore, our data include zip code for residence and not place of injury for those not injured at home. Although it is likely that falls in very young children occurring in the home were the result of window falls, among older children this is less likely as fewer than half of the falls in older adolescents occurred at home, leaving us with inadequate information with which to design or assess interventional strategies for this group. ICD-9-CMs do not provide sufficient information to enable one to tailor interventions across the adolescent age span. There is a need to improve documentation and completeness of injury coding in medical records and administrative data sets.

In summary, in New York City, where window guard legislation is enforced and exposure to multifamily dwellings is higher, injury from falls from buildings and structures is half that observed nationally. The finding that warm weather risks begin earlier in the spring and extend later in the fall season than previously reported has important prevention implications for both developing and developed societies. Window guards have potential to provide affordable, effective prevention of injuries in developing societies where the population is disproportionately young and living in warm climates, and where high rise and multiple storey buildings are being used increasingly to alleviate overcrowding.

\section{ACKNOWLEDGEMENTS}

This work was funded by the National Center for Minority Health and Health Disparities through the EXPORT grant mechanism (NIH grant numbers 1P60MD000206) and by the Robert Wood Johnson Foundation of Princeton, New Jersey. We are grateful to the New York State Department of Health for providing NY State Hospital Discharge data which allowed us to compare incidence of injury between areas with and without legislated and enforced window guard legislation. Sionery Villar assisted with an earlier version of this work.

\section{Authors' affiliations}

J C Pressley, Injury Free Coalition for Kids, Departments of Epidemiology and Health Policy and Management in the Mailman School of Public Health, Columbia University, New York, NY

B Barlow, Injury Free Coalition for Kids, Department of Surgery at Harlem Hospital, College of Physicians and Surgeons, Columbia University, New York, NY

This work was funded by the National Center for Minority Health and Health Disparities through the EXPORT grant mechanism (NIH grant numbers 1P60MD000206) and by the Robert Wood Johnson Foundation of Princeton, New Jersey.

Conflicts of interest: neither of the authors has any conflict of interest.

Ethics approval: the scope of the work included in this manuscript was approved by the Columbia University Medical Center Institutional Review Board, 722 W 168th Street, New York, NY, USA.

\section{REFERENCES}

1 Barlow B, Niemirska M, Gandhi RP, et al. Ten years of experience with falls from height in children. J Pediatr Surg 1983;18:509-11.

2 Garrettson LK, Gallagher SS. Falls in children and youth. Pediatr Clin North Am 1985;32:153-62.

3 New York City Department of Health and Mental Hygiene. Window Falls Prevention Program. Available at http://www.ci.nyc.ny.us/html/doh/html/ win/winbroc.html (accessed 15 November 2004).

4 Boston Public Health Commission. Childhood Injury Prevention Program (CIPP), Window Guards/Kids Can't Fly. Available at http://www.bphc.org/ health/atoz.asp?id = 95 (accessed 5 April 2005)

5 Bergner L, Mayer S, Harris D. Falls from heights: a childhood epidemic in an urban area. [historical article reprinted from 1971] Injury Prev 1995; 1:191-4.

6 Spiegel CN, Lindaman FC. Children can't fly: a program to prevent childhood morbidity and mortality from window falls. Am J Public Health 1977:67:1143-7.

7 Bijur PE, Spiegel C. Window fall prevention and fire safety: 20 years of experience in New York City. Pediatr Res 1996;39:102A.

8 Lehman D, Schonfeld N. Falls from heights: a problem not just in the northeast. Pediatrics 1993:92:121-4.

9 Benoit R, Watts DD, Dwyer K, et al. Windows 99: a source of suburban pediatric trauma. J Trauma 2000;49:477-81.

10 Keogh S, Gray JS, Kirk CJ, et al. Children falling from a height in London. Inj Prev 1996;2:188-91

11 Ong ME, Ooi SB, Manning PG. A review of 2,517 childhood injuries seen in a Singapore emergency department in 1999-mechanisms and injury prevention suggestions. Singapore Med J 2003;44:12-19.

12 Lallier M, Bouchard S, St-Vil D, et al. Falls from heights among children: a retrospective review. J Pediatr Surg 1999;34:1060-3.

13 Adesunkanmi AR, Oseni SA, Badru OS. Severity and outcome of falls in children. West Afr J Med 1999;18:281-5

14 Mirdad T. Fractures of the neck of femur in children: an experience at the Aseer Central Hospital, Abha, Saudi Arabia. Injury 2002;33:823-7.

15 Seleye-Fubara SD, Ekere AU. Domestic accidental deaths in the Niger Delta Region, Nigeria. East Afr Med J 2003;80:622-6.

16 Stone KE, Lanphear BP, Pomerantz WJ, et al. Childhood injuries and deaths due to falls from windows. J Urban Health 2000;77:26-33.

17 Mathis RD, Levine SH, Phifer S. An analysis of accidental free falls from height: the "spring break" syndrome. J Trauma 1993;34:123-6. 
18 American Academy of Pediatrics, Committee on Trauma. Falls from heights: windows, roofs, and balconies. Pediatrics 2001;107:1188-91.

19 Musemeche CA, Barthel M, Cosentino C, et al. 1991. Pediatric falls from heights. J Trauma 1991;31:1347-9.

20 Williams RA. Injuries in infants and small children resulting from witnessed and corroboincidenced free falls. J Trauma 1991;31:1350-2.

21 Meller JL, Shermata DW. Falls in urban children: a problem revisited. Am J Dis Child 1987; 141:1271-5.

22 Kids' Inpatient Databases Technical Documentation, Healthcare Cost and Utilization Project. Department of Health and Human Services, Agency for Healthcare Research and Quality, Rockville MD, 2000.

23 US Census Bureau. Geographic comparison tables for population, housing units, area and density: 2000. Available at http://factfinder.census.gov (accessed 9 December 2003).

24 Agency for Health Care Policy and Research. Clinical classifications for health policy research: tools for decision making and research. AHCPR Pub no. 98-0049. Rockville, US: Department of Health and Human Services, 1999.

25 Hosmer DW, Lemeshow S. Applied logistic regression. 2nd edition. New York: John Wiley and Sons, 1999.

26 SAS Institute. SAS/STAT User's guide. Version 6. 4 ed. Volume 1 and 2 . Cary, US: SAS Institute Inc, 1993.

27 Gill JR. Fatal descent from height in New York City. J Forensic Sci 2001;46:1132-7.

28 Fewer Children Falling From Windows. New York Times, 8/26/74, p33.

29 Child [aged] 3, Survives a 12-Story Fall. New York Times, 04/19/1976, pl6.

30 Cincotti JA. New York gets tough on window guard law. New York Times, 8/ 25/1987, pB3.

31 Vinici RJ, Freedman E, Wolski K. Preventing falls from windows: the efficacy of the Boston window falls prevention program. Arch Pediatr Adolesc Med $1996 ; 150: 32$.

\section{BOARD MEMBER BIOGRAPHY}

\section{Roy J Shephard}

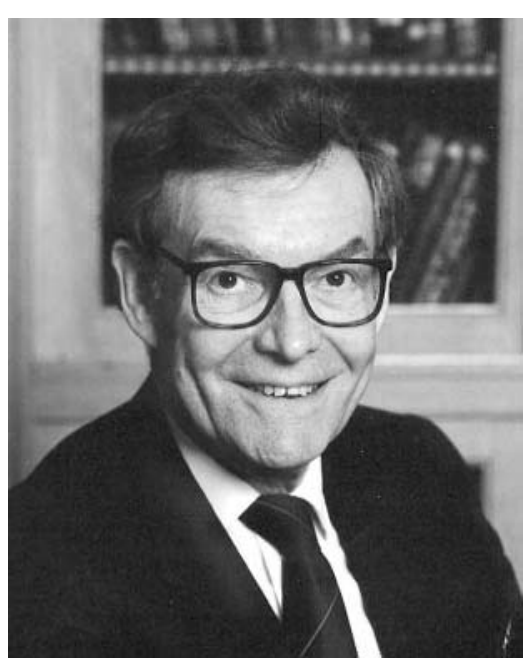

$\mathrm{R}$ oy J Shephard, MD (Lond), PhD, DPE is Professor Emeritus of Applied Physiology in the University of Toronto. After completing his training in medicine and physiology, he pursued graduate work in cardiorespirology at Guy's Hospital, University of London. He then served in the Royal Air Force High Altitude Research Unit, Farnborough, Hants, the Department of Preventive Medicine and Applied Physiology of the University of Cincinnati, and the Chemical Defence Research Establishment at Porton Down before accepting an appointment in Toronto, as Professor of Applied Physiology and Director of the newly established Physical Fitness Research Unit.

A 34 year career at the University of Toronto included a Professorship in the Department of Public Health Sciences and Directorship of the School of Physical and Health Education. He is the author or part-author of over 100 books and more than 1000 peer reviewed journal articles. His research has covered a wide range of topics in Population Health, Health Promotion, Preventive Medicine, Applied Physiology, and Exercise Immunology, including specific studies of athletic injuries. His contributions have been recognized by many honours and awards, including Honorary Degrees from the Université de Montréal and Ghent University, and the Presidency and Honour Awards from both the American College of Sports Medicine and the Canadian Association of Sports Sciences.

He has now moved to the west coast of British Columbia, where he continues his writing and editorial work for a large number of scientific journals. 